\section{Análise de timol por CLAE na tintura de Lippia sidoides Cham. (alecrim-pimenta) produzida em diferentes estágios de desenvolvimento da planta}

\author{
Leal, L.K.A.M. ${ }^{1 *}$; Oliveira, V.M. ${ }^{1}$; Araruna, S.M. ${ }^{2}$; Miranda, \\ M.C.C. ${ }^{3}$; Oliveira, F.M.A. ${ }^{3}$;
}

\begin{abstract}
${ }^{1}$ Laboratório de Farmacognosia, Departamento de Farmácia, Universidade Federal do Ceará

${ }^{2}$ Laboratório Central de Saúde Pública

${ }^{3}$ Secretaria da Saúde, Prefeitura Municipal de Fortaleza
\end{abstract}

\section{Resumo}

O teor de timol, princípio ativo usado como marcador nas tinturas das folhas de Lippia sidoides Cham. (Verbenaceae) preparadas com material coletado antes, durante e após a floração e designadas como T1, T2 e T3, foi determinado por CLAE, utilizando-se cromatógrafo Shimadzu CLASS-VP, coluna RP-18 (Supelco), com fase móvel isocrática acetonitrila:água (78:22) e fluxo $0,8 \mathrm{ml} / \mathrm{min}$ com detecção em $254 \mathrm{~nm}$. As amostras foram injetadas num volume de $20 \mu \mathrm{l}$ e analisadas em triplicata. Os teores de timol encontrados foram 1,97 $\pm 0,07 \mathrm{em} \mathrm{T1} ; 2,00 \pm$ $0,03 \mathrm{em} \mathrm{T} 2$ e 2,34 $\pm 0,06 \mathrm{mg} / \mathrm{ml} \mathrm{em} \mathrm{T3}$. Os resultados mostram discretas diferenças nas concentrações de timol nas tinturas das folhas de alecrim-pimenta preparadas em diferentes momentos de seu desenvolvimento. Contudo, observou-se que o melhor momento para a coleta da planta parece ser após a sua floração (T3), que mostrou o maior teor de timol.

\begin{abstract}
Lippia sidoides Cham. (Verbenaceae) is popularly known in Northeast Brazil as "alecrim-pimenta". The goal of the present work is to perform a quantitative analysis of timol, used as a marker in phytomedicine prepared from the leaves of $L$. sidoides, collected before, during and after the flower. At least three samples of tincture of $L$. sidoides were analyzed by HPLC. Among the samples analyzed, the tincture produced from the leaves after the flower showed slightly high concentration of timol $(2,34 \pm 0,06 \mathrm{mg} / \mathrm{ml})$ when compared with before $(1,97 \pm 0,07$ $\mathrm{mg} / \mathrm{ml})$ or during the flower $(2,00 \pm 0,03 \mathrm{mg} / \mathrm{ml})$. The best period to collect leaves of $L$. sidoides seems to be after the flower.
\end{abstract}

O taxon genérico Lippia (Verbenaceae) inclui aproximadamente 200 espécies de ervas, arbustos e de árvores de pequeno porte ${ }^{1}$. Lippia sidoides Cham. (syn. L. multicapitata Mart.) é uma planta arbustiva, aromática, que ocorre na região nordeste do Brasil com grande freqüência na área abrangida pelos municípios de Mossoró, RN e Tabuleiro do Norte (CE), onde é conhecida pelos rurícolas como alecrim-pimenta, alecrim e estrepa-cavalo. Suas folhas são utilizadas popularmente sob a forma de chá abafado ou tintura como anti-séptico local ${ }^{2,3}$.

O óleo essencial obtido das folhas de Lippia sidoides tem como principal constituinte o timol, cujo teor tem variado entre 34,2 a 95,1\% em várias determinações. Outros constituintes encontrados são o carvacrol, $p$-cimeno, $\alpha$-terpineno e $\beta$ cariofileno ${ }^{4,5,6}$. O estudo químico de extratos de Lippia sidoides levou ao isolamento e caracterização de compostos fixos, incluindo dois dímeros naftoquinônicos (lapachenolisocatalponol e tectol), ésteres metílicos naturais dos ácidos graxos de $\mathrm{C}_{16}$ a $\mathrm{C}_{24}, \beta$-sitosterol, ácido vanílico, 2-metil-5isopropilfenol e a 5-4-dihidroxi-6,7-dimetoxi-flavona ${ }^{7,8,9}$.

O ensaio toxicológico agudo pré-clínico demonstrou a baixa toxicidade dos constituintes hidrossolúveis arrastados por vapor d'água durante a extração de óleo essencial das folhas de $L$. sidoides ${ }^{10}$. Além disso, o teste de reação por contato, feito com a aplicação de óleo essencial a $1 \%$ na pele dos animais, não causou alergia ${ }^{11}$. O espectro de atividade antibacteriana e antifúngica do óleo essencial se relaciona a microorganismos como Escherichia coli, Staphylococcus aureus, Pseudomonas aeruginosa, Acinetobacter sp, Streptococcus mutans, Corynebacterium xerosis, Cândida albicans, Trichophytum rubrum e Trichophytum interdigitale, dentre outros ${ }^{5,6,12,13}$.

Existem numerosos fatores que podem interferir quantitativa e qualitativamente na produção de óleo essencial de plantas aromáticas, tendo um papel decisivo na qualidade do produto final. O rendimento e a composição podem ser influenciados pelas condições climáticas, hora da coleta, idade da planta, cuidados na preparação da matéria-prima vegetal, além de outros fatores ${ }^{14,15}$. Considerando que a influência desses fatores deve ser estudada caso a caso, desenvolveu-se este trabalho cujo objetivo foi investigar a provável variação do teor de timol na tintura das folhas de L. sidoides coletadas em diferentes momentos de seu desenvolvimento, ou seja, antes, durante e após o período de floração.

Foi observada apenas uma discreta variação nas concentrações de timol nas amostras de tintura de Lippia sidoides produzidas a partir das folhas coletadas antes, durante e depois da floração das plantas. O melhor momento para a coleta da planta parece ser após a sua floração, considerando que o teor determinado neste momento do desenvolvimento da planta foi maior do que o encontrado $(2,34 \pm 0,06 \mathrm{mg} / \mathrm{ml})$ nas demais tinturas da planta antes da floração $(1,97 \pm 0,07 \mathrm{mg} / \mathrm{ml}) \mathrm{e}$ durante a floração $(2,00 \pm 0,03 \mathrm{mg} / \mathrm{ml})$ (figura 1).

A figura 2 apresenta o perfil cromatográfico da tintura obtida da planta após a floração (T3). Os resultados observados são interessantes, considerando que Lippia sidoides permanece na floração durante quase todo o período anual, daí reduções significativas no teor de timol na planta durante essa fase poderiam interferir na qualidade dessa matéria-prima, devendose levar em conta também, no entanto, a relação risco-benefício, considerando a pequena variação do princípio ativo e o tempo de espera, do momento para a preparação de tintura ou para extração do óleo essencial ${ }^{16}$. Embora estudos anteriores não tenham sido realizados quanto à variação de timol nas folhas de Lippia sidoides durante seu ciclo vital, já foi demonstrada uma variação sazonal na planta quanto ao teor de timol, e observada também uma redução em torno de $30,7 \%$ no rendimento da extração do óleo essencial das folhas, após cinco meses de seu armazenamento $^{17}$. 
Estudos dessa natureza devem ser feitos condicionados às possíveis variáveis, a fim de conseguir dados que possam orientar o trabalho de coleta das folhas de Lippia sidoides, que são amplamente utilizadas nos programas governamentais de fitoterapia na região nordeste do Brasil.

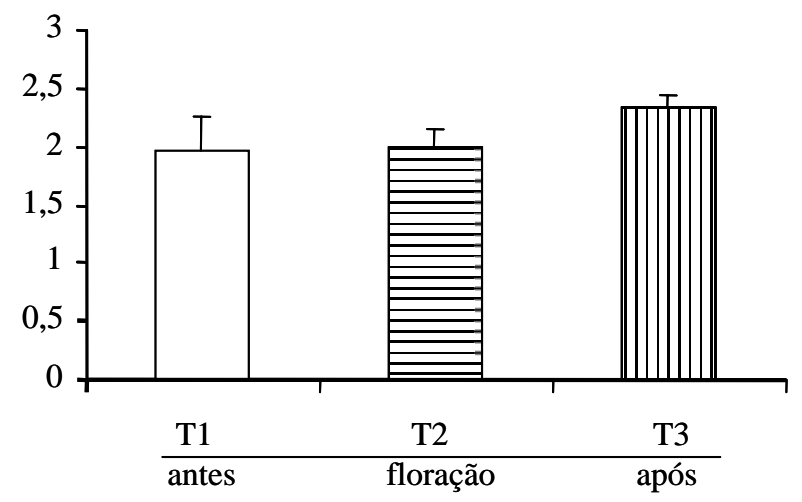

Figura 1. Teor de timol na tintura das folhas de Lippia sidoides coletadas antes, durante e após a floração. Os valores representam a média \pm d.p.m. da concentração de timol nas $\operatorname{amostras}(n=3-4)$.
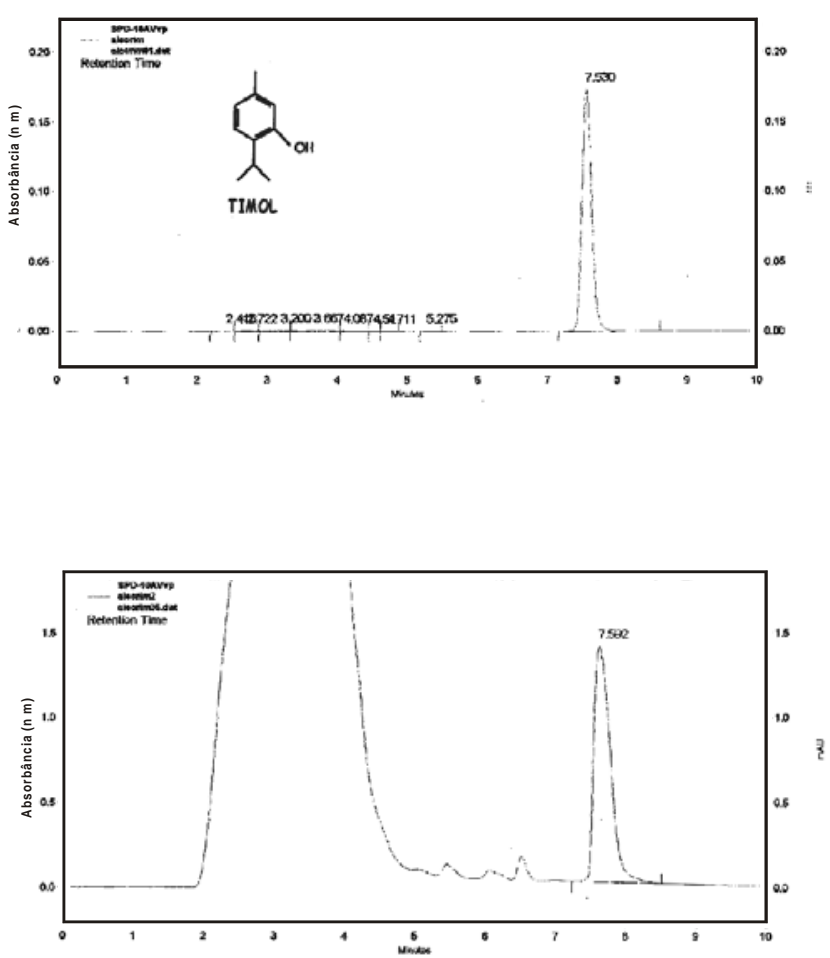

Figura 2. Cromatograma (CLAE-UV) do timol (A) e da tintura de Lippia sidoides (B) obtida das folhas coletadas após a floração.

\section{Material e Métodos}

Material botânico: As folhas de Lippia sidoides foram coletadas em Fortaleza (CE) (fevereiro-julho/2000), no horto de plantas medicinais da Universidade Federal do Ceará (UFC), no Campus do Pici. Exsicatas das mesmas plantas submetidas à coleta foram preparadas e depositadas no Herbário Prisco Bezerra da UFC, sob o número EAC-08474.

Tinturas de Lippia sidoides: Foram obtidas segundo metodologia descrita na Farmacopéia Brasileira (2a ed.). Quarenta gramas das folhas de $L$. sidoides de cada tipo foram submetidas à secagem natural (sombra), trituradas e extraídas com $200 \mathrm{ml}$ de etanol:água (70:30) durante 5 dias, à temperatura ambiente, num percolador. Cada tintura (T1, T2 e T3) foi analisada diretamente por CLAE-UV.

Análises Cromatográficas: As análises por CLAE-UV foram realizadas em cromatógrafo líquido Shimadzu CLASS-VP, coluna RP-18 (Supelco) de $250 \mathrm{~mm} \times 4 \mathrm{~mm} \times 5 \mathrm{~mm}$, temperatura $40^{\circ} \mathrm{C}$, fase móvel isocrática acetonitrila:água (78:22) e fluxo $0,8 \mathrm{ml} / \mathrm{min}$ com detecção em $254 \mathrm{~nm}$. Foram injetados $20 \mu \mathrm{l}$ de cada amostra, em triplicata ou mais, quando necessário. Para a determinação quantitativa do timol, foi desenvolvida uma curva de calibração, utilizando-se soluções de timol nas concentrações de 0,125 ;

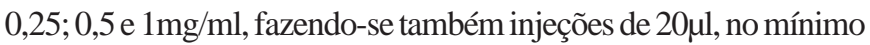
três vezes.

Agradecimentos: FUNCAP e CNPq pelo apoio financeiro.

\section{Referências}

${ }^{1}$ Terblanché, F.C.; Kornelius G. Essential oil constituents of the genus Lippia (Verbenaceae): A literature review. Journal of Essential Oil Research, v. 8, p. 471-485, 1996.

${ }^{2}$ Matos, F.J.A., Plantas Medicias: guia de seleção e emprego de plantas usadas em fitoterapia no Nordeste do Brasil . 2. ed. Fortaleza, Imprensa Universitária / UFC, 2000. p. 171-172.

3 Aguiar, L.B.M.A.; Matos, F.J.A. Atividade antibiótica de plantas da flora nordestina - II. Ciência e Cultura, v. 36, p. 464, 1983.

${ }^{4}$ Matos, F.J.A.; Oliveira, F. Lippia sidoides Cham.: farmacognosia, química e farmacologia. Rev. Bras. Farm., v. 79, p. 84-87, 1998.

${ }^{5}$ Lacoste, E.; Chaumont, J.P.; Mandin, D.; Plumel, M.M.; Matos, F.J. Antiseptic properties of essential oil of Lippia sidoides Cham. Application to the cutaneous microflora. Ann. Pharm. Fr., v. 54, p. 228-230, 1996.

${ }^{6}$ Lemos, T.L.J.; Monte, F.J.Q.; Barbosa, R.C.B.; Lima, E.O. Chemical composition and antimicrobial activity of essential oils from brazilian plants. Fitoterapia, v. 63, p. 266-268, 1992.

${ }^{7}$ Costa, S.M.O.; Lemos, T.L.G.; Monte, F.J.A. Quinona dimérica (tectol) de Lippia sidoides (Verbenaceae). In: XV Simpósio de Plantas Medicinais do Brasil, Águas de Lindóia, SP, 1998.

${ }^{8}$ Macambira, L.M.A. Contribuição ao conhecimento químico de plantas do Nordeste - Lippia sidoides Cham. Dissertação de Mestrado, UFC, 1985.

${ }^{9}$ Macambira, L.M.A.; Andrade, C.H.S.; Matos, F.J.A.; Craveiro, A.A.; Braz-Filho, R. Naphtoquinoids from Lippia sidoides. J. Nat. Prod., v. 49, p. 310-312, 1986.

${ }^{10}$ Viana, G.S.B.; Matos, F.J.A.; Craveiro, A.A. Observações preliminares sobre a farmacologia de Lippia sidoides. $30^{\mathrm{a}}$ Reunião Anual da SPBC, SP, Ciência e Cultura, v. 30, 1978.

${ }^{11}$ Mendonça, V.L.M.; Fonteles, M.C.; Aguiar, L.M.B.; Craveiro, A.A. Toxicidade e alergenicidade do óleo essencial de Lippia 
sidoides Cham. para utilização em cosméticos. Aerosois e Cosméticos, v. 12, p. 12-17, 1990.

${ }^{12}$ Aguiar, L.B.M.; Matos, F.J.A. Atividade antibiótica de plantas medicinais da flora nordestina. Ciência e Cultura, v. 35, 1983.

${ }^{13}$ Lemos, T.L.J.; Matos, F.J.A.; Matos, F.J.A.; Craveiro, A.A.; Mechesney, J.D. Antimicrobial activity of essential oils of brazilian plants. Phytoterapy Research, v. 4, p. 82-84, 1990.

${ }^{14}$ Miraldi, E.; Giachetti, D.; Ferri, S. Quality control of aromatic drugs reported in European Pharmacopoeia 3. ed., IL Fármaco, v. 56, p. 365-371, 2001.

${ }^{15}$ Silva, M.G.V.; Craveiro, A.A.; Matos, F.J.A.; Machado, M.I.L.; Alencar, J.W. Chemical variation during daytime of constituents of the essential oil of Ocimum gratissimum leaves. Fitoterapia, v. 70, p. 32-34, 1999.

${ }^{16}$ Smith, R.M. Gas and liquid chromatography in analytical chemistry. Chichester: Jonh Wiley, 1988.

${ }^{17}$ Nunes, R.S. Desenvolvimento galênico de produtos de uso odontológico (creme dental e enxaguatório bucal) à base de Lippia sidoides Cham. - Verbenaceae -alecrim-pimenta. Dissertação de Mestrado, UFPE, 1999.

*Autor para correspondência:

Luzia Kalyne A. M. Leal

Rua Valdemiro Cavalcante, 340 - Rodolfo Teófilo

CEP 60 430-050 - Fortaleza (CE)

E-mail: kalyne@ufc.br
Anatomia foliar de Bauhinia blakeana Dunn.

Ferreira, J.L.P. ${ }^{1,2}$; Velasco, E. ${ }^{1}$; Paula, A.E.S. ${ }^{1}$; Araújo, R.B. ${ }^{1}$; Pacheco, J.M. ${ }^{2}$

1 Laboratório de Química de Produtos Naturais, FarManguinhos, Fundação Oswaldo Cruz

${ }^{2}$ Laboratório de Farmacognosia, Departamento de Farmácia e Administração Farmacêutica, Faculdade de Farmácia, Universidade Federal Fluminense

\section{Resumo}

São observadas as características microscópicas das folhas de Bauhinia blakeana Dunn., espécie conhecida no Brasil como "Pata de vaca" e usada pela população como hipoglicemiante. As análises dos cortes transversais e das dissociações epidérmicas indicam a presença de um mesofilo dorsiventral contendo espaços intercelulares, protegido por epidermes com tricomas de diversos aspectos e estômatos paracíticos e anomocíticos. O pecíolo, plano-convexo, pubescente, apresenta duas saliências, colênquima angular e feixes vasculares colaterais e bicolaterais.

\begin{abstract}
The paper describes the microscopic characteristics of the leaves of Bauhinia blakeana Dunn., known in Brazil as "pata de vaca" and used as a hypoglycemic remedy. Transverse sections and epidermal dissociations indicate the presence of a dorsiventral mesophyll protected by epidermis with trichomes of diverse types and paracytic and anomocytic stomata. The petiole, which is plane-convex, pubescent, shows two excrescences, an angular collenchyma and collateral and bicollateral vascular bundles.
\end{abstract}

Bauhinia blakeana Dunn. (LeguminosaeCaesalpinioideae) (figura 2-J), árvore muito solicitada como ornamental pelo porte e beleza de suas flores, foi descrita como uma nova espécie em 1908, após ser descoberta no litoral da ilha de Hong Kong, por volta do ano de $1880^{1}$, sendo considerada por alguns autores como híbrido de B. variegata e B. purpurea. Ao ser trazida para o Brasil adaptou-se plenamente às condições climáticas e edáficas locais. Hoje encontra-se cultivada principalmente na região sudeste brasileira, onde é conhecida pelo nome de "pata de vaca" e considerada possuir, como outras espécies próximas, que recebem a mesma designação nominal, propriedades hipoglicemiantes. A população brasileira costuma 